\title{
Feasibility of the Use of RapiGraft and Skin Grafting in Reconstructive Surgery
}

\author{
Jung Dug Yang, In Gook Cho, Joon Hyun Kwon, Jeong Woo Lee, Kang Young Choi, \\ Ho Yun Chung, Byung Chae Cho \\ Department of Plastic and Reconstructive Surgery, Graduate School of Medicine, Kyungpook National University, Daegu, Korea
}

Background Skin grafting is a relatively simple and thus widely used procedure. However, the elastic and structural quality of grafted skin is poor. Recently, various dermal substitutes have been developed to overcome this disadvantage of split-thickness skin grafts. The present study aims to determine the feasibility of RapiGraft as a new dermal substitute.

Methods This prospective study included 20 patients with partial- or full-thickness skin defects; the patients were enrolled between January 2013 and March 2014. After skin defect debridement, the wound was divided into two parts by an imaginary line. Split-thickness skin grafting alone was performed on one side (group A), and RapiGraft and split-thickness skin grafting were used on the other side (group B). All patients were evaluated using photographs and self-questionnaires. The Manchester scar scale (MSS), a chromameter, and a durometer were used for the scar evaluation. The average follow-up period was 6 months.

Results The skin graft take rates were $93 \%$ in group $A$ and $89 \%$ in group $B$, a non-significant difference $(P=0.082)$. Statistically, group $B$ had significantly lower MSS, vascularity, and pigmentation results than group $A(P<0.05$ for all). However, the groups did not differ significantly in pliability $(\mathrm{P}=0.155)$.

Conclusions The present study indicates that a simultaneous application of RapiGraft and a split-thickness skin graft is safe and yields improved results. Therefore, we conclude that the use of RapiGraft along with skin grafting will be beneficial for patients requiring reconstructive surgery.

Keywords Skin / Artificial / Cicatrix / Skin transplantation
Correspondence: Jung Dug Yang Department of Plastic and Reconstructive Surgery, Graduate School of Medicine, Kyungpook National University, 130 Dongdeokro, Jung-gu, Daegu 41944, Korea Tel: +82-53-420-5688 Fax: +82-53-425-3879 E-mail: lambyang@daum.net

This work was presented at the 72nd Congress of the Korean Society of Plastic and Reconstructive Surgeons on November 9, 2014, in Seoul, Korea.

No potential conflict of interest relevant to this article was reported.

\section{INTRODUCTION}

Skin grafting procedures comprise the classical reconstruction method for the coverage of full-thickness defects due to trauma, burns, or tumor excision. As full-thickness skin grafting involves the entire epidermis and dermis, such methods are reliable but limited in scale. Split-thickness skin grafting is commonly performed because this procedure has few range limitations and in- volves a relatively simple operation. However, split-thickness skin grafts are complicated by poor quality at the recipient site in terms of skin structure and elasticity, as well as blood circulation, and are often aesthetically unsatisfactory and result in scar contracture $[1,2]$.

Recently, various dermal substitutes have been developed to overcome the shortcomings of split-thickness skin grafts and obtain more satisfactory results. Among these, Integra (Integra 
Life Science Corporation, Plainsboro, NJ, USA) has been proven useful in various clinical studies [3-5]. However, Integra has the disadvantage of a necessary second-stage surgical treatment to remove the upper silicon-based component 2-3 weeks after the initial application to the skin defect [6]. Subsequently, Oasis (Cook Biotech, West Lafayette, IN, USA), a collagen matrix derived from porcine small intestinal submucosa; Alloderm ( $\mathrm{KCI}$ Life Cell Corp., San Antonio, TX, USA), derived from cadavers; and Matriderm (Dr. Suwelack Skin and Health Care AG, Billerbeck, Germany), which is extracted from bovine collagen, were developed and have been used widely to date. These dermal substitutes enhance the skin quality and yield enhanced functional and aesthetic results, but have certain disadvantages.

RapiGraft (DalimTissen Co., Seoul, Korea) is a very pure form of Type I collagen that is extracted from porcine skin. This dermal substitute features a cross-linked single-layered membrane with superior physical stability as compared to non-cross-linked matrices, and is thus beneficial for skin structure reconstruction. In addition, this material can be easily produced. However, no objective evaluation of RapiGraft has been reported thus far. Accordingly, the present study was conducted to examine the usefulness of this dermal substitute.

\section{METHODS}

Protocol approval for this prospective study was obtained from the Institutional Review Board (KNUH IRB's approval: 201304-080) prior to the initiation of the study. A total of 20 patients with partial- or full-thickness skin defects (12 men, 8 women; average age, 48 years; range, $29-82$ years) with causes including

\section{Fig. 1. Study protocol}

After debridement was performed on the defective skin area, one group of patients received only split-thickness skin grafts (STSG) (group A) whereas the other group received simultaneous rapigraft and split-thickness skin grafts (group B).

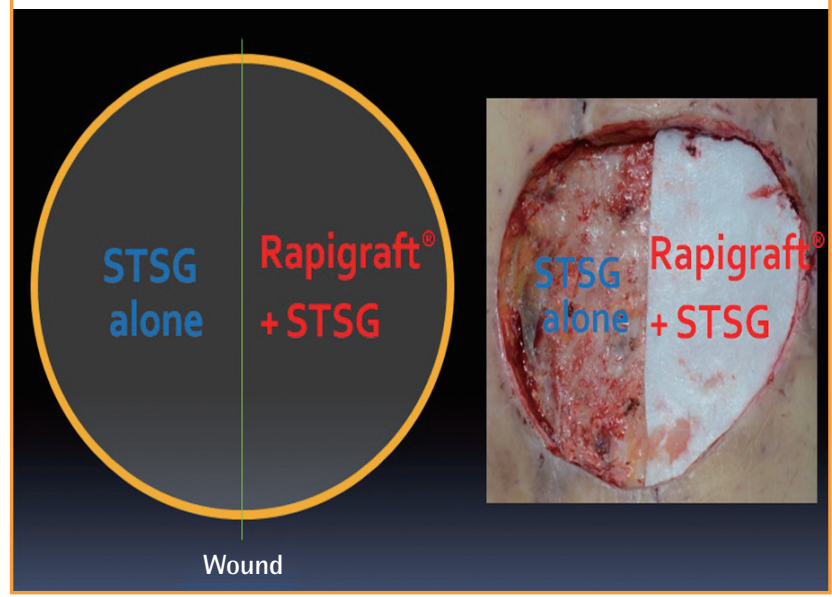

traffic accidents, tumor excision, and diabetes mellitus were enrolled between January 2013 and March 2014. Informed consent was obtained from the legal agents of all patients. The average follow-up period for all these patients was 6 months.

After skin defect debridement, the wound was divided into two parts by an imaginary line. Split-thickness skin grafting alone was performed on one side (group A), and RapiGraft and splitthickness skin grafting were used on the other side (group B) (Fig. 1). The grafts were fixed using nylon and staples, and engraftment was promoted using a tie-over dressing to prevent hematoma. For wound stabilization, a splint was applied to render the graft site immovable. Patients received inpatient treatment until the wound healed completely. All patients underwent 6 months of follow-up observation, during which a photographic evaluation was conducted at 3, 5, 7, and 14 days and 1, 3, and 6 months after the operation. The skin grafting take rate was evaluated through photography on day 14 after the operation by three plastic surgeons, using ACDsee Pro 7 (ACD Systems, Seattle, WA, USA) in order to reduce bias. The take rate was also evaluated via digital photography by measuring the ratio of the number of grids in the viable areas to the number of grids in the totally grafted areas (Fig. 2).

In month 6, skin quality was evaluated using the Manchester Scar Scale, and patients were also asked to self-estimate their satisfaction through questionnaires. The Manchester Scar Scale was initially presented by Beausang et al. in 1998 as a method of scar evaluation according to characteristics such as color, con-

\section{Fig. 2. Skin graft take rate evaluation}

The take rate was determined from digital photographs as the ratio of the number of grids in the viable areas to the number of grids in the totally grafted areas. STSG, splith thickness skin graft.

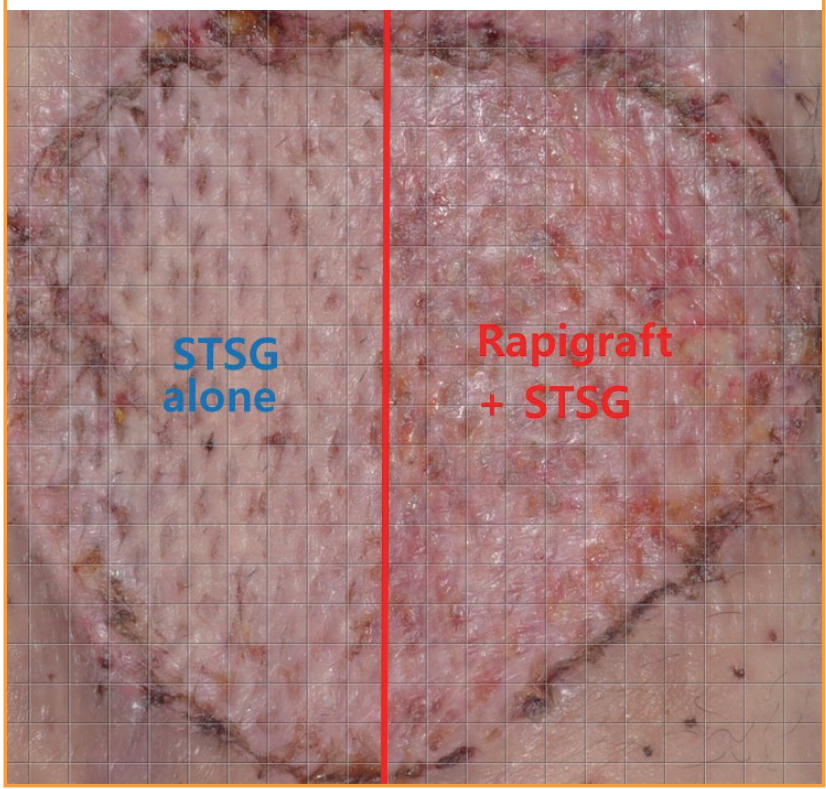


tour, texture, appearance, and distortion. In this scale, a lower score indicates a higher skin quality [7]. A chromameter (CR400, Minolta, Tokyo, Japan), which quantitatively measures skin vascularity and pigmentation, and a durometer (Rex Gauge Company Inc., Glenview, IL, USA), which quantitatively measures skin firmness, were also used for representing the items evaluated by the Manchester Scar Scale objectively. Differences between groups A and B and normal skin were thus measured, compared, and evaluated [8]. To reduce bias, three plastic surgeons measured each evaluation tool; the measured values were then averaged. A patient satisfaction survey was conducted in month 6 by using a visual analog scale; as with the Manchester Scar Scale, a lower score indicated a higher level of satisfaction. A statistical analysis was conducted using a paired t-test (IBM SPSS Statistics ver. 22.0, IBM Corp., Armonk, NY, USA).

\section{RESULTS}

A total of 20 patients underwent treatment and follow-up as prescribed in the original treatment plan. The causes of skin defects were diverse, although the majority of the defects were caused by crush injuries from traffic accidents; other causes included skin tumors, flap donor sites, and necrotizing fasciitis. The grafted areas were mainly the extremities (Table 1). Treatment success was generally observed on day 14 after the operation, with grafted skin take rates of $93 \% \pm 5.6 \%$ and $89 \% \pm 5.8 \%$ in groups A and B, respectively; this difference was not statisti- cally significant $(\mathrm{P}=0.082)$ (Fig. 3 ). In most of the considered cases, RapiGraft and a skin graft were successfully applied simultaneously. The results of the RapiGraft application are presented in Figs. 4 and 5.

The Manchester Scar Scale scores were 11.2 \pm 3.1 and $7.9 \pm 2.3$ in groups A and B, respectively. With respect to vascularity, the chromameter yielded values of $10.4 \pm 2.3$ and $5.6 \pm 1.6$ for groups $\mathrm{A}$ and $\mathrm{B}$, respectively, with the corresponding pigmentation values of $6.6 \pm 0.8$ and $3.5 \pm 0.5$, respectively. With respect to firmness, the durometer yielded values of $4.4 \pm 1.2$ and $3.9 \pm 1.1$ for groups $\mathrm{A}$ and $\mathrm{B}$, respectively, and the corresponding self-satisfaction evaluation results were $4.3 \pm 1.2$ and $2.9 \pm 1.3$, respectively (Table 2). Statistically, group B had significantly higher

\section{Fig. 3. Take rates at day 14 after surgery}

Represented as the percentage of the viable area to the entire area. No statistically significant difference was observed $(P=0.082)$. STSG, splith thickness skin graft.

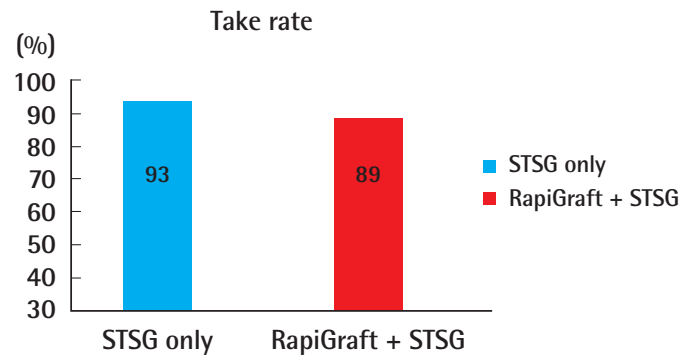

\section{Table 1. Patient characteristics}

\begin{tabular}{|c|c|c|c|c|c|c|c|}
\hline Patient no. & Age (yr) & Sex & Indication & Recipient site & Timing & Defect & Skin type \\
\hline 1 & 59 & Male & Bicycle TA & Medial aspect of the upper arm & Delayed & Full & Mesh \\
\hline 2 & 30 & Male & Motorcycle TA & Lower $1 / 3$ of the leg & Delayed & Full & Mesh \\
\hline 3 & 60 & Male & Pedestrian TA & Lower $1 / 3$ of the leg & Delayed & Partial & Mesh \\
\hline 4 & 72 & Female & Necrotizing fasciitis & Thigh & Delayed & Full & Mesh \\
\hline 5 & 87 & Male & Pedestrian TA & Lower $1 / 3$ of the leg & Delayed & Full & Mesh \\
\hline 6 & 44 & Male & Pedestrian TA & Medial aspect of the upper arm & Delayed & Full & Mesh \\
\hline 7 & 75 & Male & DM ulcer & Foot dorsum & Delayed & Full & Mesh \\
\hline 8 & 78 & Male & Flap donor site & Forearm & Immediate & Full & Sheet \\
\hline 9 & 33 & Female & Pedestrian TA & Lower $1 / 3$ of the leg & Delayed & Full & Sheet \\
\hline 10 & 33 & Female & Pedestrian TA & Lower $1 / 3$ of the leg & Delayed & Full & Sheet \\
\hline 11 & 68 & Male & Pedestrian TA & Thigh & Delayed & Full & Sheet \\
\hline 12 & 91 & Male & Necrotizing fasciitis & Thigh & Delayed & Full & Mesh \\
\hline 13 & 53 & Female & Phlebitis & Foot dorsum & Delayed & Full & Mesh \\
\hline 14 & 79 & Female & Liposarcoma & Forearm & Immediate & Full & Sheet \\
\hline 15 & 87 & Male & Bicycle TA & Foot dorsum & Delayed & Full & Mesh \\
\hline 16 & 75 & Female & Motorcycle TA & Lower $1 / 3$ of the leg & Delayed & Full & Mesh \\
\hline 17 & 78 & Female & Pedestrian TA & Lower $1 / 3$ of the leg & Delayed & Full & Sheet \\
\hline 18 & 33 & Male & Necrotizing fasciitis & Foot dorsum & Delayed & Full & Mesh \\
\hline 19 & 33 & Male & Pedestrian TA & Forearm & Delayed & Full & Mesh \\
\hline 20 & 68 & Female & Pedestrian TA & Lateral aspect of the upper arm & Delayed & Partial & Mesh \\
\hline
\end{tabular}




\section{Fig. 4. Case 1}

(A) Preoperative view, the defect after a pedestrian traffic accident of the medial aspect of the upper arm. (B) Intraoperative view, split-thickness skin grafting alone on one side, and RapiGraft and split-thickness skin grafting on the other side. (C) Postoperative view at 2 weeks of follow-up, the take rate of both sides was good. (D) Postoperative view at 6 months of follow-up, an aesthetically pleasant result.
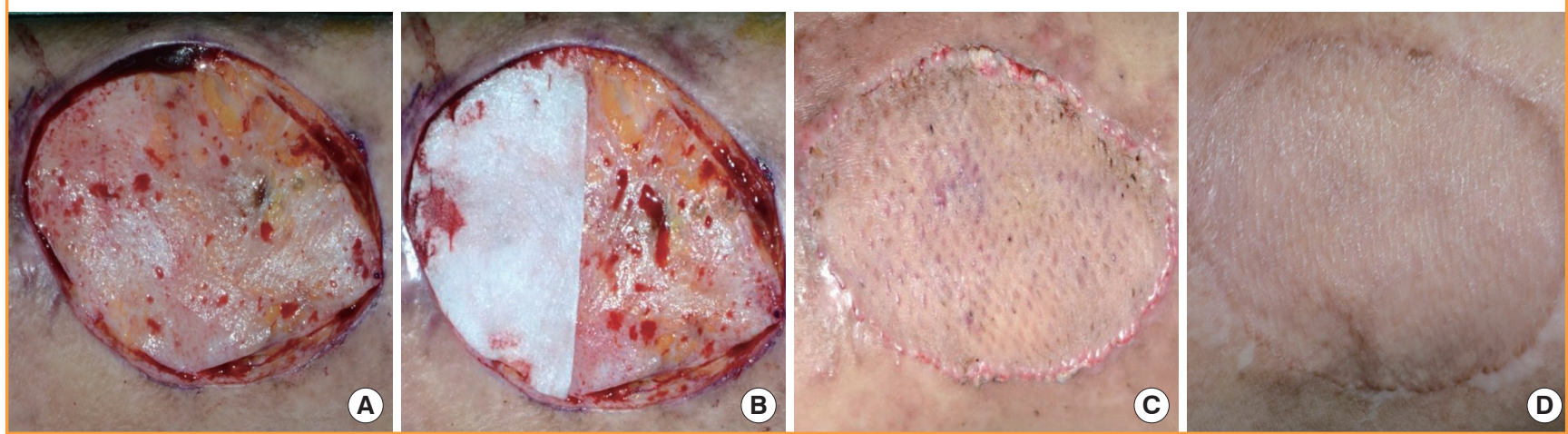

\section{Fig. 5. Case 2}

(A) Preoperative view, the defect after a bicycle traffic accident of the foot dorsum. (B) Intraoperative view, split-thickness skin grafting alone was performed on one side, and RapiGraft and split-thickness skin grafting on the other side. (C) Postoperative view at 2 weeks of follow-up, the take rate of both sides was good. (D) Postoperative view at 6 months of follow-up, an aesthetically pleasant result.
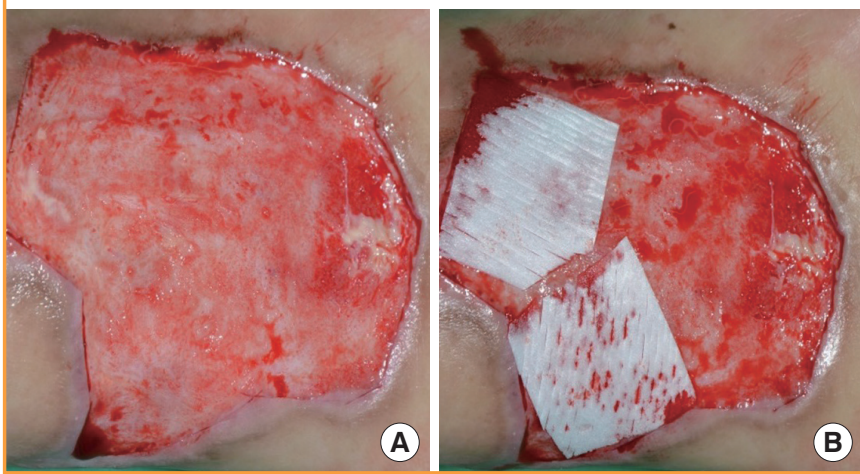
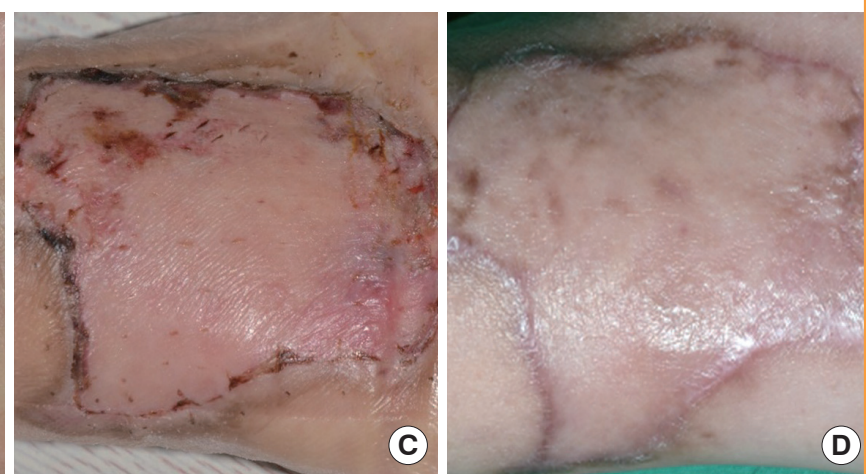

Table 2. Graft outcome evaluation parameters

\begin{tabular}{|lrcr|}
\hline Scar scale & $\begin{array}{c}\text { STSG only } \\
\text { (group A) }\end{array}$ & $\begin{array}{c}\text { RapiGraft+STSG } \\
\text { (group B) }\end{array}$ & P-value \\
\hline Vascularity & $10.4 \pm 2.3$ & $5.6 \pm 1.6$ & $<0.001$ \\
Pigmentation & $6.6 \pm 0.8$ & $3.5 \pm 0.5$ & $<0.001$ \\
Firmness & $4.4 \pm 1.2$ & $3.9 \pm 1.1$ & 0.155 \\
Self-estimation & $4.3 \pm 1.2$ & $2.9 \pm 1.3$ & 0.012 \\
Manchester scar scale & $11.2 \pm 3.1$ & $7.9 \pm 2.3$ & $<0.001$ \\
\hline STSG, splith thickness skin graft. & & \\
\hline
\end{tabular}

skin quality enhancement values than did group A for all parameters except the durometer-measured firmness, and significantly higher patient satisfaction $(\mathrm{P}<0.05$ for all except durometermeasured firmness).

\section{DISCUSSION}

Artificial skin substitutes have been developed to overcome the unsatisfactory cosmetic results of skin grafting, which result from disadvantageous factors such as reduced skin elasticity, skin structure degradation, and excessive scar formation. According to previous research conducted by van Zuijlen et al. [9], the results of large-scale, long-term follow-up observations of short-term clinical studies revealed no statistical evidence of the long-term clinical effects of artificial skin materials; however, many studies have reported survey results demonstrating that artificial skin substitutes increase the quality of skin grafts and comprise easy-to-perform reconstruction methods $[10,11]$. Accordingly, Integra, Oasis, Alloderm, and Matriderm were developed and remain widely used today.

Unlike Integra, which requires a two-stage operation, RapiGraft does not require an additional operation and is thus convenient for the patient; in addition, in Korea, RapiGraft is less expensive than Matriderm and can thus be used by more patients. Additionally, RapiGraft is easier to produce than Alloderm, which uses cadavers and materials with an irregular supply 
and demand.

RapiGraft is made of a highly pure form of Type I atelocollagen, extracted from porcine skin, from which telopeptide, which causes an immune reaction with proteolytic enzymes, has been removed. Notably, the use of porcine rather than bovine collagen eliminates the risk of transmissible spongiform encephalopathy $[12,13]$. This wound-coating material features a cross-linked, single-layered membrane and superior mechanical strength, with a slower decomposition rate as compared to that of Matriderm. The cross-linked nature of the RapiGraft matrix is thought to exhibit superior physical stability as compared to a noncross-linked matrix, which might better facilitate skin structure reconstruction [14]. Moreover, in contrast to the cytotoxicity induced by Integra, which is cross-linked with glutaraldehyde, 1-ethyl-3-(3-dimethylaminopropyl)carbodiimide (EDC), which is used in RapiGraft, is converted into a water-soluble urea form and exhibits very low cytotoxicity $[15,16]$.

Our study, similar to other studies, showed that reconstruction is possible via a one-stage surgical treatment without any engraftment failure in the areas of skin and soft tissue loss. In this study, we did not observe what are often considered disadvantages of artificial skin substitutes, such as an increased nutrient and oxygen transport distance to the graft due to the insertion of artificial skin substitutes and a reduced engraftment rate [17]. Although a difference was observed in the take rates of groups $\mathrm{A}$ and $B$, this difference was not statistically significant. Accordingly, our results were similar to those of studies on artificial skin substitutes conducted by Hansbrough et al. [18] and Ryssel et al. [19].

In the present study, group B, to which artificial dermal substitutes and skin grafts were applied simultaneously, exhibited enhanced skin quality according to the Manchester Scar Scale, which is commonly used for evaluating skin quality. Moreover, differences between groups $\mathrm{A}$ and $\mathrm{B}$ and normal skin were numerically confirmed using a chromameter and a durometer. The vascularity and pigmentation values in group $\mathrm{B}$ were more similar to those of normal skin, which objectively proves that RapiGraft application enhanced skin quality. These results were similar to those from a study on Matriderm [17], and from an evaluation of scars of post-traumatic wounds to which Matriderm had been applied [20]. Taken together, these results prove the usefulness of RapiGraft.

However, there was no significant difference between the firmness results of the durometer in the two groups. This is attributed to the high inter- or intra-observer variability, one of the disadvantages of the durometer [8]. Moreover, in the scar scale results reported by Ryssel et al. [19]. For sheet-type skin grafts, the use of a dermal matrix yielded a clear increase in elasticity, whereas no differences were observed with mesh-type skin grafts, although this might have been due to the lack of distinction in the research method used by the authors.

A considerable drawback with respect to RapiGraft is that the follow-up observation period is less than 1 year, and as a result, long-term follow-up observations are needed. Thus, RapiGraft needs to be applied and evaluated in additional patients and more diverse areas of joints with exposed bones and tendons.

According to the results of this research, the simultaneous use of RapiGraft and a split-thickness skin graft can safely heal wounds and enhance skin quality such as that of scar scales. Moreover, RapiGraft showed results similar to those of other skin substitutes and is cost-efficient, so it is useful for patients suffering from skin defects who need skin grafting for prevention of the cicatricial contracture and for enhancement of the skin quality. Thus, it can be a reliable option to skin grafting.

\section{REFERENCES}

1. Haslik W, Kamolz LP, Manna F, et al. Management of fullthickness skin defects in the hand and wrist region: first longterm experiences with the dermal matrix Matriderm. J Plast Reconstr Aesthet Surg 2010;63:360-4.

2. Yannas IV, Burke JF. Design of an artificial skin: I. Basic design principles.J Biomed Mater Res 1980;14:65-81.

3. Lamme EN, de Vries HJ, van Veen H, et al. Extracellular matrix characterization during healing of full-thickness wounds treated with a collagen/elastin dermal substitute shows improved skin regeneration in pigs. J Histochem Cytochem 1996;44:1311-22.

4. Groos N, Guillot M, Zilliox R, et al. Use of an artificial dermis (Integra) for the reconstruction of extensive burn scars in children: about 22 grafts. Eur J Pediatr Surg 2005; 15:18792.

5. King WW, Lam PK, Liew CT, et al. Evaluation of artificial skin (Integra) in a rodent model. Burns 1997;23 Suppl 1: S30-2.

6. Burke JF, Yannas IV, Quinby WC Jr, et al. Successful use of a physiologically acceptable artificial skin in the treatment of extensive burn injury. Ann Surg 1981;194:413-28.

7. Beausang E, Floyd H, Dunn KW, et al. A new quantitative scale for clinical scar assessment. Plast Reconstr Surg 1998; 102:1954-61.

8. Fearmonti R, Bond J, Erdmann D, et al. A review of scar scales and scar measuring devices. Eplasty 2010;10:e43.

9. van Zuijlen PP, Vloemans JF, van Trier AJ, et al. Dermal substitution in acute burns and reconstructive surgery: a subjective and objective long-term follow-up. Plast Reconstr Surg 
2001;108:1938-46.

10. van Zuijlen PP, van Trier AJ, Vloemans JF, et al. Graft survival and effectiveness of dermal substitution in burns and reconstructive surgery in a one-stage grafting model. Plast Reconstr Surg 2000;106:615-23.

11. Wainwright D, Madden M, Luterman A, et al. Clinical evaluation of an acellular allograft dermal matrix in full-thickness burns. J Burn Care Rehabil 1996;17:124-36.

12. Song E, Yeon Kim S, Chun T, et al. Collagen scaffolds derived from a marine source and their biocompatibility. Biomaterials 2006;27:2951-61.

13. Pati F, Adhikari B, Dhara S. Isolation and characterization of fish scale collagen of higher thermal stability. Bioresour Technol 2010;101:3737-42.

14. Bottcher-Haberzeth S, Biedermann T, Schiestl C, et al. Matriderm ${ }^{\circledR} 1 \mathrm{~mm}$ versus Integra ${ }^{\circledR}$ Single Layer $1.3 \mathrm{~mm}$ for onestep closure of full thickness skin defects: a comparative experimental study in rats. Pediatr Surg Int 2012;28:171-7.

15. Park SN, Park JC, Kim HO, et al. Characterization of porous collagen/hyaluronic acid scaffold modified by 1-ethyl-3-(3dimethylaminopropyl)carbodiimide cross-linking. Biomaterials 2002;23:1205-12.

16. Sun HW, Feigal RJ, Messer HH. Cytotoxicity of glutaraldehyde and formaldehyde in relation to time of exposure and concentration. Pediatr Dent 1990;12:303-7.

17. Ryssel H, Germann G, Kloeters O, et al. Dermal substitution with Matriderm ${ }^{\circledR}$ in burns on the dorsum of the hand. Burns 2010;36:1248-53.

18. Hansbrough JF, Dore C, Hansbrough WB. Clinical trials of a living dermal tissue replacement placed beneath meshed, split-thickness skin grafts on excised burn wounds. J Burn Care Rehabil 1992;13:519-29.

19. Ryssel H, Gazyakan E, Germann G, et al. The use of MatriDerm in early excision and simultaneous autologous skin grafting in burns: a pilot study. Burns 2008;34:93-7.

20. Cervelli V, Brinci L, Spallone D, et al. The use of MatriDerm ${ }^{\circledR}$ and skin grafting in post-traumatic wounds. Int Wound J 2011;8:400-5. 